\title{
The Effect of Electroacupuncture on Neuronal Apoptosis and Related Functions in Rats with Acute Spinal Cord Injury
}

\author{
Liang Zhang1, Changming Li1 ${ }^{1}$, Renfu Quan2* , Shangju Xie ${ }^{1}$ \\ ${ }^{1}$ Research Institute of Orthopedics, Zhejiang Chinese Medical University, Hangzhou, China \\ ${ }^{2}$ Department of Orthopedics, Xiaoshan Traditional Chinese Medical Hospital, Hangzhou, China \\ Email: "quanrenfu@126.com
}

Received 23 September 2014; revised 25 October 2014; accepted 8 November 2014

Academic Editor: Qingshan (Bill) Fu, Harvard Medical School, USA

Copyright (C) 2014 by authors and Scientific Research Publishing Inc.

This work is licensed under the Creative Commons Attribution International License (CC BY). http://creativecommons.org/licenses/by/4.0/

(c) (i) Open Access

\begin{abstract}
Objective: To investigate the effect and significance of electroacupuncture (EA) on neuronal apoptosis and hindlimb motor and bladder functional improvement in rats with acute spinal cord injury (SCI). Methods: Sixty healthy Sprague Dawley rats were randomly assigned to sham, model, EA, and EA control groups ( $n=15$ each). EA group rats received EA treatment at Zhibian and Shuidao acupoints seven times daily, whereas EA control group rats received EA at two points, $0.5 \mathrm{~cm}$ away from Zhibian and Shuidao, respectively. Histomorphological changes in spinal cord tissue were examined using hematoxylin-eosin staining. Neuronal apoptosis was detected by TUNEL assay. Bcl-2, Bax, and Bad protein levels were detected using immunohistochemistry. Additionally, hindlimb motor function, residual urine volume and maximum bladder capacity were measured. $R e$ sults: HE staining revealed a morphologically and structurally intact spinal cord in the EA group, and the tissue contained scattered blood cells without edema. In the EA control group, there were small morphological defects in the spinal cord, and the tissue contained fewer blood cells with local edema. Compared with the EA control and model groups, Bax and Bad levels were significantly decreased in the EA group and Bcl-2 expression was increased $(P<0.05)$. After SCI, hindlimb function scores, residual urine volume, and maximum bladder capacity in rats of the EA group significantly differed from those of the EA control group $(P<0.05)$. Conclusion: EA may induce SCI-induced improvements in hindlimb motor and bladder functions by affecting neuronal apoptosis and relevant gene expression changes.
\end{abstract}

\footnotetext{
"Corresponding author.
}

How to cite this paper: Zhang, L., Li, C.M., Quan, R.F. and Xie, S.J. (2014) The Effect of Electroacupuncture on Neuronal Apoptosis and Related Functions in Rats with Acute Spinal Cord Injury. Chinese Medicine, 5, 199-210. 
Keywords

Electroacupuncture, Spinal Cord Injury, Neuronal Apoptosis, Molecular Mechanism

\section{Introduction}

Spinal cord injury (SCI) is a serious threat to human health. It often causes paraplegia and quadriplegia, leading to a series of serious complications and sequelae, such as urinary tract infection, respiratory tract infection, renal function impairment, and bedsores [1] [2]. SCI can substantially affect quality of life and family life situations, making it an urgent, worldwide problem to be addressed in the field of medicine. In recent years, there have been an increasing number of studies focused on the treatment, rehabilitation, and mechanisms of acute SCI. It is currently believed that mechanical injury to the spinal cord itself destroys its continuity and integrity. Following primary injury, the body initiates secondary injury to the spinal cord, leading to changes in the microenvironment, such as ischemia and hypoxia, inflammation, intracellular and extracellular calcium imbalance, excessive production of free radicals, excitatory amino acid changes, cytotoxic substances, and apoptosis, which are counterproductive to spinal cord functional recovery.

Electroacupuncture (EA) is an important part of traditional Chinese medicine, with a dual therapeutic effect of acupuncture and electrostimulation. EA stimulation at certain acupoints promotes Qi and blood circulation via meridian vessels to help regulate yin and yang and strengthen resistance to pathogenic factors. Previous acupuncture studies have focused on the analgesic, antispastic, and antidepressant effects [3]. Apoptosis is an important biological process, which involves a series of gene-regulated, initiative, cell death processes. Apoptosis exists in a number of diseases, such as Alzheimer's disease, Parkinson's disease, and traumatic injury [4] [5]. However, abnormal apoptosis can cause drastic deterioration in a variety of neurodegenerative diseases. Previous studies have documented the role of neuronal apoptosis and apoptotic gene expression in SCI [6]. In addition, Bcl-2 family proteins are key regulators of apoptosis, among which the proportion of Bcl-2, Bad, and Bax expression is the "molecular switch" to initiate apoptosis [7] [8]. It has been shown that down-regulation of Bcl-2 expression and up-regulation of Bax expression are non-conducive to the survival of hippocampal neurons [9]. A number of reports document that EA has a curative effect on neurodegenerative diseases, such as SCI [10] [11], although the underlying mechanisms remain unclear. The present study investigated the effect of EA treatment at Zhibian and Shuidao acupoints on neuronal apoptosis in rats with SCI. We further examined relevant changes in Bad, Bax and Bcl-2 expression to explore the possible molecular mechanisms of EA for promoting neuronal functional improvement after SCI.

\section{Materials and Methods}

\subsection{Experimental Animals and Grouping}

A total of 60 healthy, adult, male Sprague Dawley (SD) rats (220 - $250 \mathrm{~g}$ body weight) were purchased from the Experimental Animal Center of Xiamen University, Fujian Province, China (License No.: SYXK (Fujian) 20130006). After 1 week of adaptive feeding, the rats were randomly assigned to the sham, model, EA, and EA control groups ( $n=15$ each). Animals were sacrificed at $7 \mathrm{~d}$ post-surgery, and a 0.5 -cm-long tissue sample was taken from above and below the injured spinal cord segment for further testing.

\subsection{Reagents and Instruments}

Bcl-2, Bax, and Bad were purchased from Bioworld (Louis Park, MN, USA). The TUNEL kit was purchased from Beijing Zhongshan Reagent Company (Beijing, China). Disposable Hwato acupuncture needles and an electroacupuncture device were purchased from Suzhou Medical Appliance Factory (Jiangsu Province, China). The fixative was prepared with $4 \%$ paraformaldehyde in phosphate-buffered saline (PBS).

\subsection{Animal Model Establishment}

An SD rat model with moderate SCI was established using a modified Allen's method [12] [13]. Rats were 
fasted for $8 \mathrm{~h}$, and individual rats then underwent surgery in a random order. All groups were anesthetized via an intraperitoneal injection of $10 \%$ chloral hydrate $(0.3 \mathrm{~mL} / 100 \mathrm{mg})$ and then fixed in a prone position. An approximately 2.5 -cm-long, median, chest-back incision was aseptically made, through which the skin and subcutaneous tissues were dissected layer by layer to expose one vertebral body in the upper and lower positions, respectively. The T9-T10 spinous processes and complete vertebral plate were gouged to expose the 0.8-mm-wide spinal dura mater. A 10-g Kirschner wire was allowed to freely fall along a scaled catheter from a height of 60 $\mathrm{mm}$, which hit a 4-mm-diameter, 2-mm-wide semicircle of thin, plastic material. The object was immediately removed, thereby inducing moderate injury to the posterior horn of the spinal cord. The surrounding tissues and skin were sutured layer by layer postoperatively using 4-0 silk. The entire surgery was performed at $37^{\circ} \mathrm{C} \pm$ $0.5^{\circ} \mathrm{C}$. Animals were intraperitoneally administered 800 million $\mathrm{U} / \mathrm{d}$ penicillin postoperatively daily to prevent infection. Individuals were fed in separate cages at a room temperature of $20^{\circ} \mathrm{C}-25^{\circ} \mathrm{C}$ and supplied with adequate food and water. The bladder was massaged twice daily using Crede's method [14] [15] to help void urine until the reestablishment of reflex bladder emptying. Specific criteria were assigned for the success of model establishment: following initial injury, the injured spinal cord segment showed bleeding and edema; the rat exhibited a tail-wagging reflex with retraction-like fluttering of both lower extremities and the body; and there was flaccid paralysis in both lower extremities upon return of consciousness after anesthesia.

\subsection{Intervention Strategy}

After successful model construction, the model and sham groups remained untreated. The EA group underwent EA treatment upon return of consciousness after anesthesia. The rat was fixed within a custom-made rat bag. Acupoints were chosen according to international standard acupoints developed by the World Health Organization. Zhibian (BL54) was located in the lower hip, i.e., at the joint of outer and middle 1/3 intervals of the connection between the greater trochanter and sacral vertebra-coccygeal vertebra junction. Shuidao (ST28) was located in the abdomen, approximately $2 \mathrm{~cm}$ away from the third equal interval above the pubic symphysis on the midline between pubic symphysis and xiphosternal symphysis (divided into 13 equal parts) (Figure 1). Skin at the acupoints was prepared and disinfected. Stainless steel 0.25 -mm-diameter acupuncture needles were used to punctuate the acupoints to 4 - $5 \mathrm{~mm}$ deep, followed by $1 \mathrm{~min}$ of twirling and $15 \mathrm{~min}$ of retaining. The ipsilateral Zhibian and Shuidao points were connected to a JL2B electrical pulse stimulator to form a loop, and 2/100 $\mathrm{Hz}$ frequency and $1 \mathrm{~mA}$ current were applied for $15 \mathrm{~min}$ of stimulation. The EA treatment alternated left and right daily. For the EA control group, treatment was performed as described for the EA group at two points $0.5 \mathrm{~cm}$ away for Zhibian and Shuidao, respectively.

\subsection{Evaluation Indices and Methods}

\subsubsection{Behavioral and Motor Function Scoring}

Following model establishment, the motor function in rats of different groups was scored using the Basso, Beattie, and Bresnahan locomotor rating scale (BBB scale). SCI rats were allowed free activity in an open space. Because of limited hindlimb mobility, the rat was unable to support its body, and the hindlimbs and buttocks dragged on the ground. Rats scoring $>2$ points were considered to be failed model establishment and were subsequently excluded from the experimental group. Rats were later supplemented to these groups. BBB scoring was performed prior to model establishment, as well as immediately and $7 \mathrm{~d}$ after model establishment to ex-

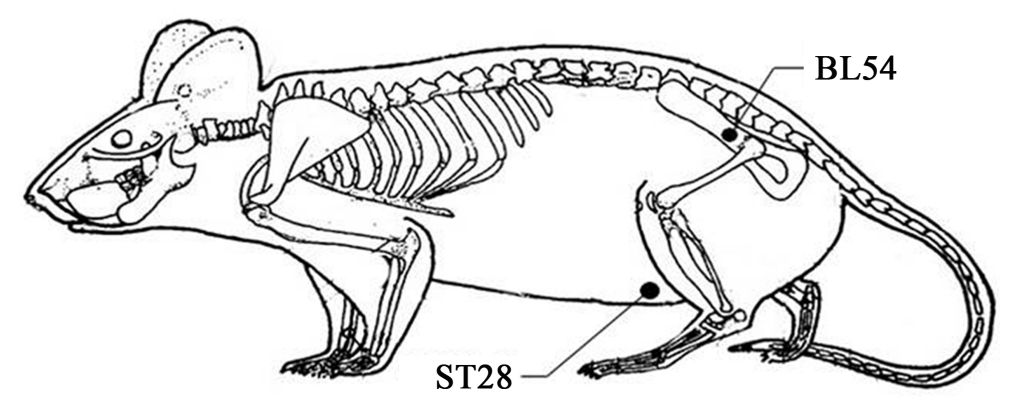

Figure 1. Schematic showing the Zhibian and Shuidao acupoints in the rat. 
amine hindlimb motor functional recovery following acute SCI. Scoring criteria included the number of movable hindlimb joints, motor coordination, and fine motor joint function. Scores were divided into 21 grades. The higher the score, the better the recovery of motor function.

\subsubsection{Residual Urine Volume Measurement}

At 1 and $7 \mathrm{~d}$ post-surgery, residual urine volume was measured by manual expression of the bladder. When held in an upright position, rats often exhibited automatic micturition. After the rat supported itself and voided urine, forefingers of both hands were used to squeeze three times from the abdomen to the pelvis. During this process, pre-weighed absorbent papers were placed underneath. Following manual expression, the absorbent papers were weighed again. Increase in the weight of papers (g) was taken as the residual urine volume (mL), because the density of urine is close to that of water, $\rho \approx 1$.

\subsubsection{Maximum Bladder Capacity Measurement}

Maximum bladder capacity was measured at 1 and $7 \mathrm{~d}$ post-surgery. The rat was fixed in a supine position on a custom-made board. Iodophor was used to disinfect the external urethral orifice and anus. Two catheters were filled with sterile saline to remove the contained gas prior to use. Following lubrication with paraffin oil, one sterilized F3 urinary catheter was slowly inserted into the urethra and bladder (4.0 - $5.0 \mathrm{~cm}$ depth) via the external urethral orifice. The second F3 catheter was then lubricated with paraffin oil and slowly inserted along the end of the first catheter to the deeper depth. Crede's method was used to void urine. Once the residual urine was completely voided from the bladder through the catheter, sterile saline was slowly injected into the bladder perfusion catheter with a 1-mL syringe. The other discharger catheter placed on the rat board was subjected to close observation, and perfusion was stopped when liquid overflowed. The total volume of injected saline solution was recorded as the bladder's maximum functional capacity.

\subsubsection{Spinal Cord Tissue Sampling}

At $7 \mathrm{~d}$ after model establishment, the rats were anesthetized via intraperitoneal injection with $10 \%$ chloral hydrate $(0.3 \mathrm{~mL} / 100 \mathrm{~g})$. After thoracotomy, intubation was performed from the left ventricle to the aorta, and the right atrial appendage was then cut. The tissue was perfused with $150 \mathrm{~mL} 9 \%$ normal saline and then fixed with $250 \mathrm{~mL} 40 \mathrm{~g} / \mathrm{L}$ paraformaldehyde. After $2 \mathrm{~h}$, a 0.5-cm-long spinal cord tissue was taken from above and below the injured segment, respectively. The specimens were fixed in the same fixative for another $24 \mathrm{~h}$, followed by conventional paraffin embedding. Serial sections ( $\sim 6 \mathrm{~m}$ thick) were prepared for hematoxylin-eosin (HE) staining, terminal deoxyribonucleotidyl transferase-mediated dUTP nick-end labeling (TUNEL) assay, and immunohistochemical (IHC) analysis.

\subsubsection{HE Staining}

HE staining was performed on three tissue sections randomly selected from each rat in different groups. After dewaxing, the sections were rinsed with double-distilled water for $1 \mathrm{~min}$ and stained with hematoxylin for 10 min. Excess dye was removed with double-distilled water, and specimens were immersed in $1 \%$ hydrochloric alcohol for $1 \mathrm{~min}$ of color separation (to avoid over-separation). Following treatment with an alkaline pro-blue solution, the sections were rinsed with flowing water and then counterstained with eosin for 2 min, dehydrated with graded ethanol (50\%, 70\%, 80\%, 90\%, and 100\%), clarified using xylene, and mounted with neutral gum. Changes in the structure and morphology of spinal cord tissues were examined under an Olympus optical microscope.

\subsubsection{TUNEL Detection of Apoptotic Cells}

Three tissue sections from each rat were dewaxed and digested with proteinase K (0.02\% mass fraction) for 30 $\mathrm{min}$. The sections were fixed in $40 \mathrm{~g} / \mathrm{L}$ paraformaldehyde and then incubated with drops of horseradish peroxidase at $37^{\circ} \mathrm{C}$ for $30 \mathrm{~min}$. Finally, diaminobenzine (DAB) was used for coloration, followed by counterstaining with lighter hematoxylin, dehydration, and mounting. The number of positive cells was quantified, and the apoptotic index $(\mathrm{AI})=$ number of apoptotic nuclei/total number of nuclei.

\subsubsection{IHC Staining}

Three tissue sections from each rat were used to detect Bcl-2, Bad, and Bax expression with an SP kit following 
the manufacturer's instructions. After conventional dewaxing, the sections were processed with $3 \%$ hydrogen peroxide, followed by high-temperature restoration and serum blocking. The sections were incubated with drops of primary antibody (dilution factor $1: 150$ ) at $4^{\circ} \mathrm{C}$ overnight. The negative control was prepared with PBS substituting the primary antibody. On the following day, secondary antibody was dropped onto the sections and incubated for $20 \mathrm{~min}$, followed by incubation with strept avidin-biotin complex (SABC) at $37^{\circ} \mathrm{C}$ for $20 \mathrm{~min}$. Thereafter, DAB was added for coloration and the color development background was examined by microscopy. Between the above steps, sections were rinsed three times with $0.01 \mathrm{~mol} / \mathrm{L}$ PBS for $5 \mathrm{~min}$ each. Finally, the sections were dehydrated with gradient alcohol, clarified with xylene, and mounted with neutral resin. Morphological changes in spinal cord tissue were examined under a light microscope.

\subsection{Statistical Analysis}

Data are presented as mean \pm standard deviation $(\bar{x} \pm \mathrm{s})$. For normally distributed data with equal variances, comparison of group means was performed using one-way analysis of variance with the LSD and SNK methods. For normally distributed data with unequal variances, the Tamhane T2 and Dunnett T3 methods were used for variance test and pairwise comparison. Data that did not follow a normal distribution were analyzed using the rank-sum test. Statistical analysis was performed using SPSS13.0 (SPSS, Chicago, IL, USA).

\section{Results}

\subsection{Behavioral Observations and BBB Scores}

Prior to surgery, all groups had normal BBB scores. Immediately post-surgery, only the sham group had a BBC score of 21, whereas the remaining three groups had the same BBC score of 0 , indicating successful model establishment. Compared with the model group, the EA and EA control groups had no significant difference in BBC score immediately or $1 \mathrm{~d}$ post-surgery $(P>0.05)$. However, BBC scores were significantly greater in the latter two groups than in the model group at 3 and $7 \mathrm{~d}$ post-surgery $(P<0.05)$. Compared with the EA control group, the EA group had significantly increased BBC scores at $7 \mathrm{~d}$ post-surgery $(P<0.05)$ (Table 1$)$.

\subsection{Residual Urine Volume}

Residual urine volume was measured in rats at $1 \mathrm{~d}$ post-surgery and remeasured after $7 \mathrm{~d}$ of treatment. Compared with the model group, the EA and EA control groups had no significant difference in residual urine volume at $1 \mathrm{~d}$ post-surgery $(P>0.05)$. However, the residual urine volume of rats was significantly less in the EA group compared with the model group at $7 \mathrm{~d}$ post-surgery $(P<0.05)$. The decrease in residual urine volume of the EA group was more significant when compared with the EA control group at $7 \mathrm{~d}$ post-surgery $(P<0.05)$ (Table 2).

\subsection{Maximum Bladder Capacity}

Maximum bladder capacity was measured in rats at $1 \mathrm{~d}$ post-surgery and remeasured after $7 \mathrm{~d}$ of treatment. There was no significant difference in maximum bladder capacity of rats in the EA and EA control groups compared with the model group at $1 \mathrm{~d}$ post-surgery $(P>0.05)$. However, maximum bladder capacity was significantly less in the EA group compared with the model group at $7 \mathrm{~d}$ post-surgery $(P<0.05)$. The decrease in maximum bladder capacity was more significant in the EA group compared with the EA control group at $7 \mathrm{~d}$ post-surgery $(P<0.05)$ (Table 3$)$.

Table 1. Comparison of BBB scores at different time points after spinal cord injury in different groups of rats $(\bar{x} \pm \mathrm{s})$.

\begin{tabular}{|c|c|c|c|c|}
\hline \multirow{2}{*}{ Group } & \multicolumn{4}{|c|}{ BBB score } \\
\hline & Immediately post-surgery & $1 \mathrm{~d}$ post-surgery & $3 \mathrm{~d}$ post-surgery & 7 d post-surgery \\
\hline Model & $0 \pm 0$ & $0.6 \pm 0.52$ & $1.9 \pm 0.74$ & $3.9 \pm 1.20$ \\
\hline EA & $0 \pm 0$ & $0.7 \pm 0.53$ & $2.9 \pm 1.10^{\amalg \square}$ & $7.5 \pm 1.58^{m \boldsymbol{\bullet}}$ \\
\hline EA control & $0 \pm 0$ & $0.8 \pm 0.52$ & $2.7 \pm 0.95^{\square}$ & $5.3 \pm 1.95^{\square m}$ \\
\hline Sham & $21 \pm 0$ & $21 \pm 0$ & $21 \pm 0$ & $21 \pm 0$ \\
\hline
\end{tabular}

Note: EA, electroacupuncture; 3 and 7 d post-surgery, compared with the model group, $\boldsymbol{m} P<0.05$; and 7 d post-surgery, compared with the EA control group, ${ }^{\bullet P} P<0.05$. 
Table 2. Comparison of residual urine volume at different time points after spinal cord injury in different groups of rats $(\bar{x} \pm s)$.

\begin{tabular}{|c|c|c|c|}
\hline \multirow{2}{*}{ Group } & \multirow[t]{2}{*}{ Number of rats } & \multicolumn{2}{|c|}{ Residual urine volume (mL) } \\
\hline & & $1 \mathrm{~d}$ post-surgery & $7 \mathrm{~d}$ post-surgery \\
\hline Model & 15 & $2.02 \pm 0.20$ & $1.41 \pm 0.07$ \\
\hline EA & 15 & $1.99 \pm 0.15$ & $1.14 \pm 0.09$ \\
\hline EA control & 15 & $2.00 \pm 0.16$ & $1.23 \pm 0.06^{\mathbf{m}}$ \\
\hline
\end{tabular}

Note: EA, electroacupuncture; 7 d post-surgery, compared with the model group, "' $P<0.05$; and $7 \mathrm{~d}$ post-surgery, compared with the EA control group, ${ }^{\bullet P} P<0.05$.

Table 3. Comparison of maximum bladder capacity at different time points after spinal cord injury in different groups of rats $(\bar{x} \pm s)$.

\begin{tabular}{cccc}
\hline \multirow{2}{*}{ Group } & Number of rats & \multicolumn{2}{c}{ Maximum bladder capacity $(\mathrm{mL})$} \\
\cline { 2 - 4 } & & 1 d post-surgery & 7 d post-surgery \\
\hline Sham & 15 & $0.93 \pm 0.06$ & $0.94 \pm 0.09$ \\
Model & 15 & $3.16 \pm 0.14$ & $1.67 \pm 0.07$ \\
EA & 15 & $2.95 \pm 0.11$ & $2.79 \pm 0.12^{-\bullet}$ \\
EA control & 15 & $2.89 \pm 0.13$ & $2.65 \pm 0.11^{\mathbf{m}}$ \\
\hline
\end{tabular}

Note: EA, electroacupuncture; 7 d post-surgery, compared with the model group, ${ }^{-} P<0.05$; and compared with the EA control group, $\bullet P<0.05$.

\subsection{HE Staining}

Light microscopy revealed histological changes in rat spinal cord tissue specimens of different groups. In the sham group (Figure 2(A)), the spinal cord displayed intact morphology and structure; gray matter neurons exhibited normal morphology and uniform distribution, with normal cell membrane, nucleus, and interstitial spaces; and white matter fibers were evenly distributed, with intact myelin sheath in an orderly arrangement.

In the model group (Figure 2(B)), the spinal cord appeared incomplete with defects of nerve tissue; the tissue presented with severe bleeding in the presence of a large number of blood cells; tissue was loose and had edema, while cells exhibited vacuolar degeneration with some karyopyknosis; nerve fibers were dissolved and missing; there was a decreased number of gray matter neurons, with neuronal swelling, karyorrhexis; the extracellular matrix exhibited a vacuolated pattern; and white matter fibers were decreased, unevenly distributed, and demyelinated, showing incomplete morphology with mutual integration.

In the EA group (Figure 2(C)), the morphology and structure of the spinal cord were generally intact, and the tissue contained scattered blood cells without edema; gray matter neurons exhibited normal morphology and vacuolar degeneration was alleviated; vacuolar degeneration still existed in some cells and the nuclei showed no significant pyknosis; and white matter fibers were evenly distributed and appeared morphologically intact without demyelination.

In the EA control group (Figure 2(D)), the spinal cord had a small amount of defects and the tissue contained fewer blood cells, with local edema; there were slightly less gray matter neurons, some of which still presented with vacuolar degeneration and karyopyknosis; and white matter fibers were slightly decreased and unevenly distributed, some of which were demyelinated (Figure 2).

\subsection{TUNEL Data}

TUNEL data showed that apoptosis was widely present in the spinal cord tissues. The number of TUNEL-positive cells was significantly increased in the model group. By comparison, EA treatment substantially reduced the number of TUNEL-positive cells $(P<0.01)$. The reduction in TUNEL-positive cell number was significant in the EA group compared with the EA control group $(P<0.05)$, indicating that EA treatment at Zhibian and Shuidao significantly reduced neuronal apoptosis in the spinal cord after injury (Table 4, Figure 3). 

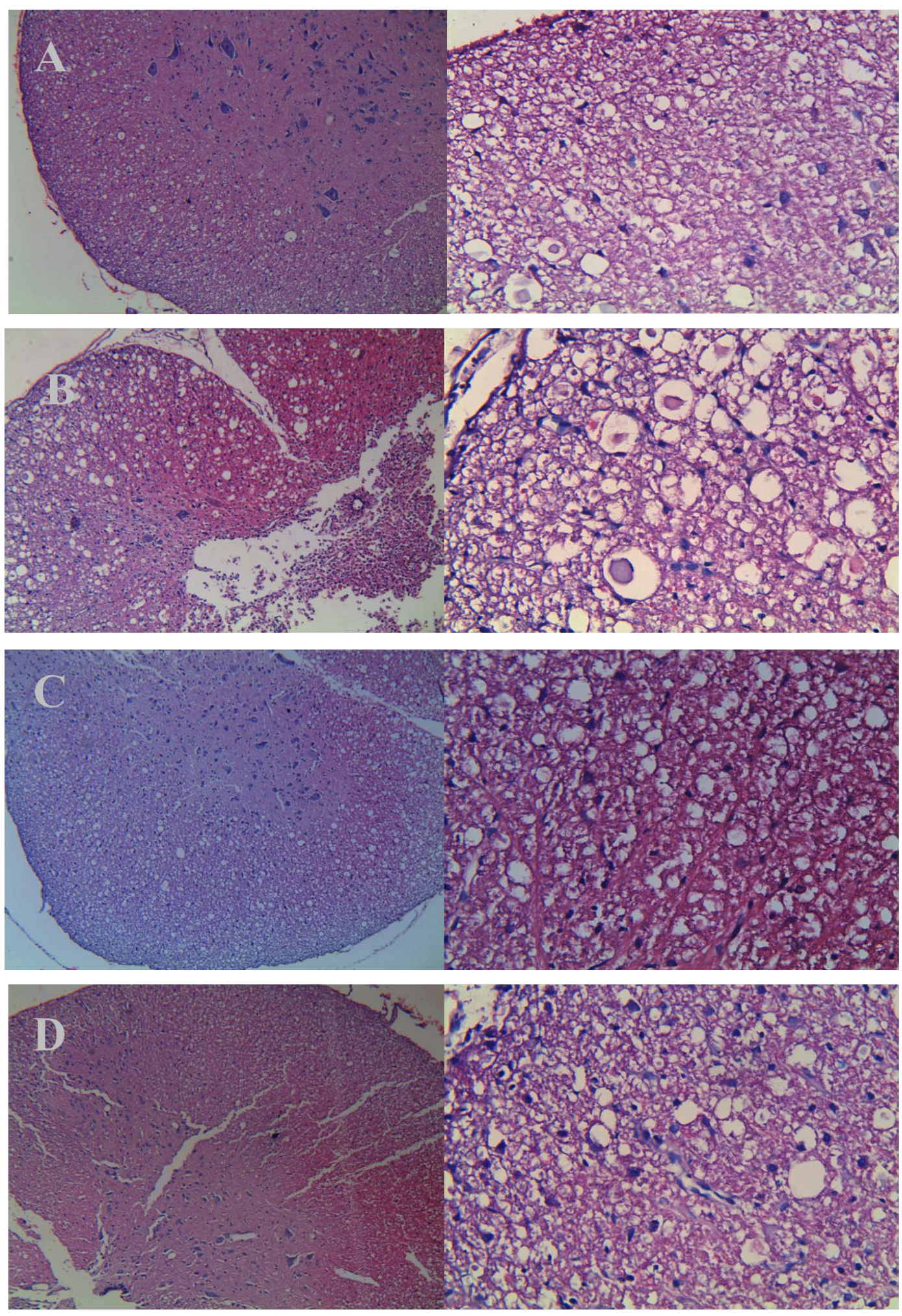

Figure 2. Microscopic observations of HE staining in injured spinal cord tissue specimens from rats of different groups at $7 \mathrm{~d}$ post-surgery (left, $\times 100$, and right, $\times 400$ for each group). (A) Sham group; (B) Model group; (C) Electroacupuncture (EA) group; and (D) EA control group. After spinal cord injury, the spinal cord showed incomplete morphology, with nervous tissue defects. After EA treatment, the spinal cord displayed intact morphology and structure, significantly better than those in the model and EA control groups. 

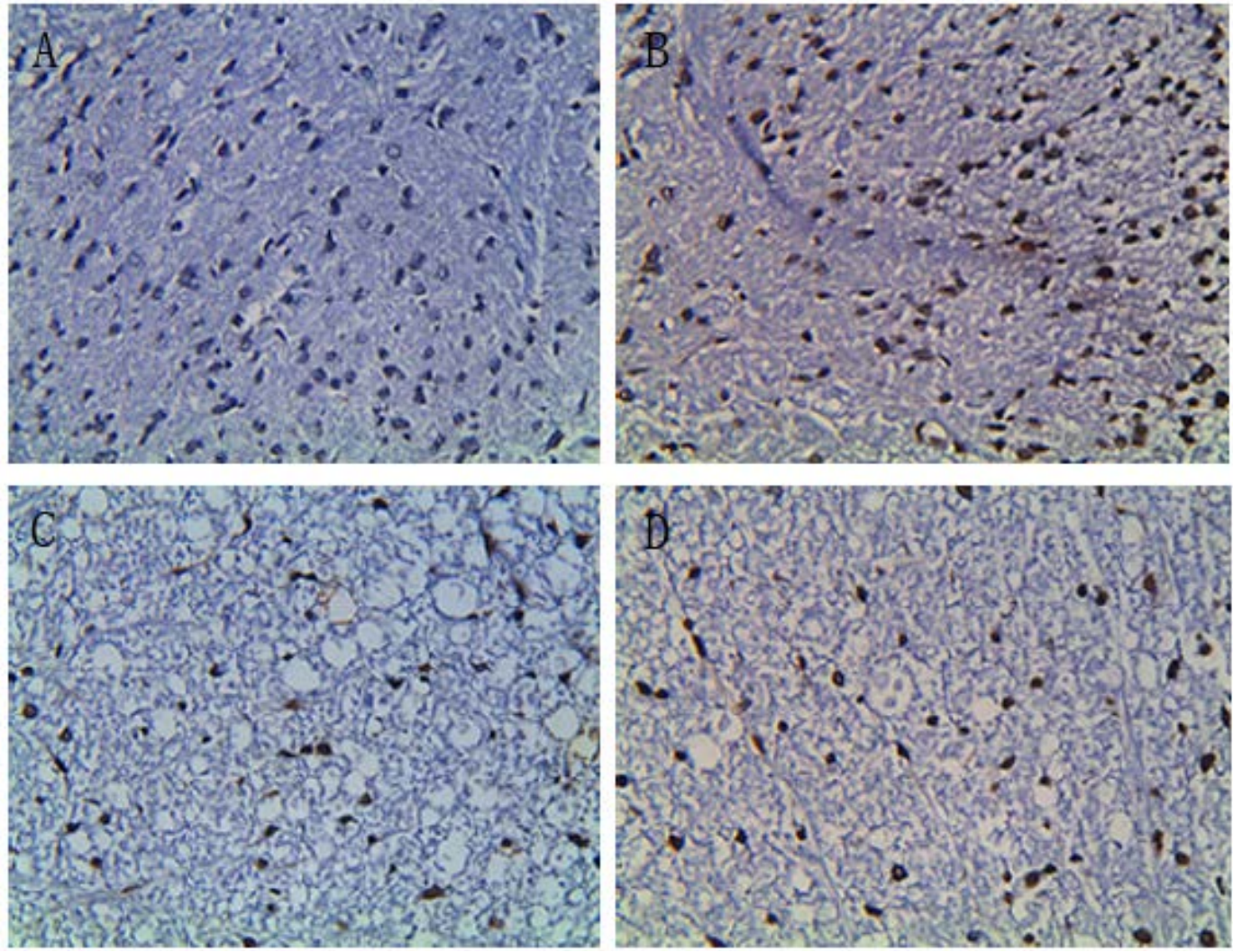

Figure 3. Microscopic observations of TUNEL staining in injured spinal cord tissue specimens from rats of different groups at $7 \mathrm{~d}$ post-surgery $(\times 100)$. (A) Sham group; (B) Model group; (C) Electroacupuncture (EA) group; and (D) EA control group. After spinal cord injury, spinal cord neurons showed different degrees of apoptosis, as well as proliferating gliocytes. The EA group had fewer apoptotic neurons, indicating that EA treatment inhibited apoptosis and promoted spinal cord injury repair. There were more apoptotic neurons in the spinal cord tissue of the EA control group, indicating that EA treatment at Zhibian and Shuidao acupoints was more effective.

Table 4. The results of TUNEL assay on injured spinal cord tissue at $7 \mathrm{~d}$ post-surgery in different groups of rats $(\bar{x} \pm s)$.

\begin{tabular}{ccc}
\hline Group & Number of rats & Apoptotic index \\
\hline Sham & 15 & $0.161 \pm 0.11$ \\
Model & 15 & $0.953 \pm 0.02$ \\
EA & 15 & $0.691 \pm 0.06^{\star \star \bullet}$ \\
EA control & 15 & $0.838 \pm 0.04^{\bullet \bullet}$
\end{tabular}

Note: EA, electroacupuncture; compared with the model group, ${ }^{\star \star} P<0.05,{ }^{\bullet \bullet} P<0.05$; and compared with the EA control group, ${ }^{\bullet \bullet} P$ $<0.05$.

\subsection{IHC Staining}

Positive IHC staining presented as a brown color. At $7 \mathrm{~d}$ after SCI, the number of Bcl-2-positive cells significantly decreased, but the number of Bax- and Bad-positive cells increased. After Zhibian and Shuidao EA treatment, the number of Bcl-2-positive cells significantly increased, while the number of Bax- and Bad-positive cells significantly decreased. These quantitative changes were statistically significant between the EA and EA control groups $(P<0.05)$, indicating that EA treatment at both acupoints and non-acupoints promoted Bcl-2 expression and inhibited Bax and Bad expression, but the regulatory effect was more significant in the EA group (Table 5, Figure 4). 
Bax
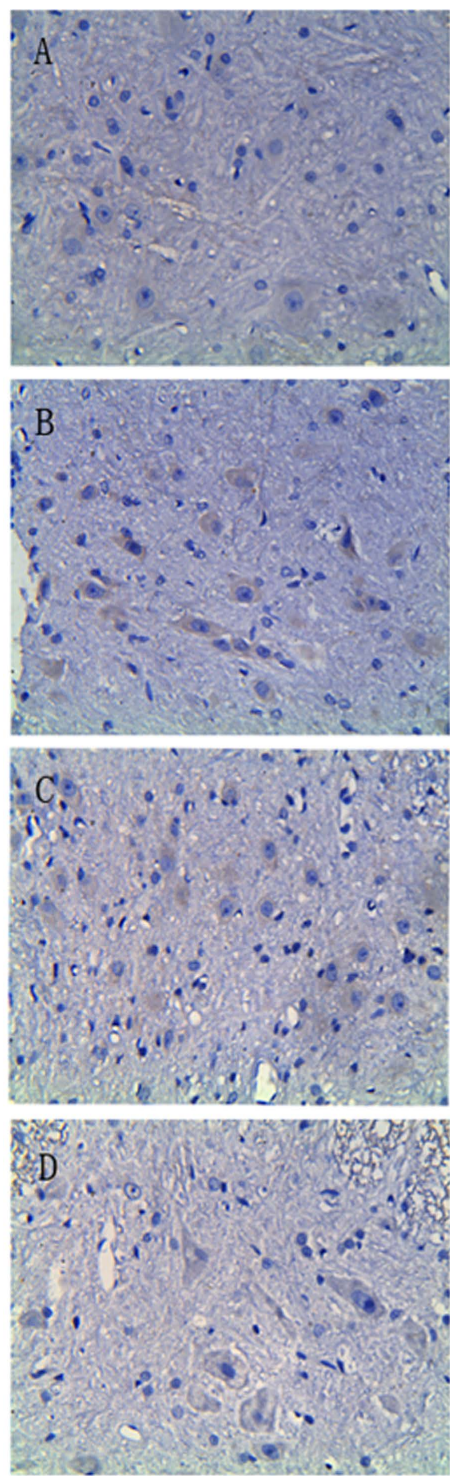

Bad
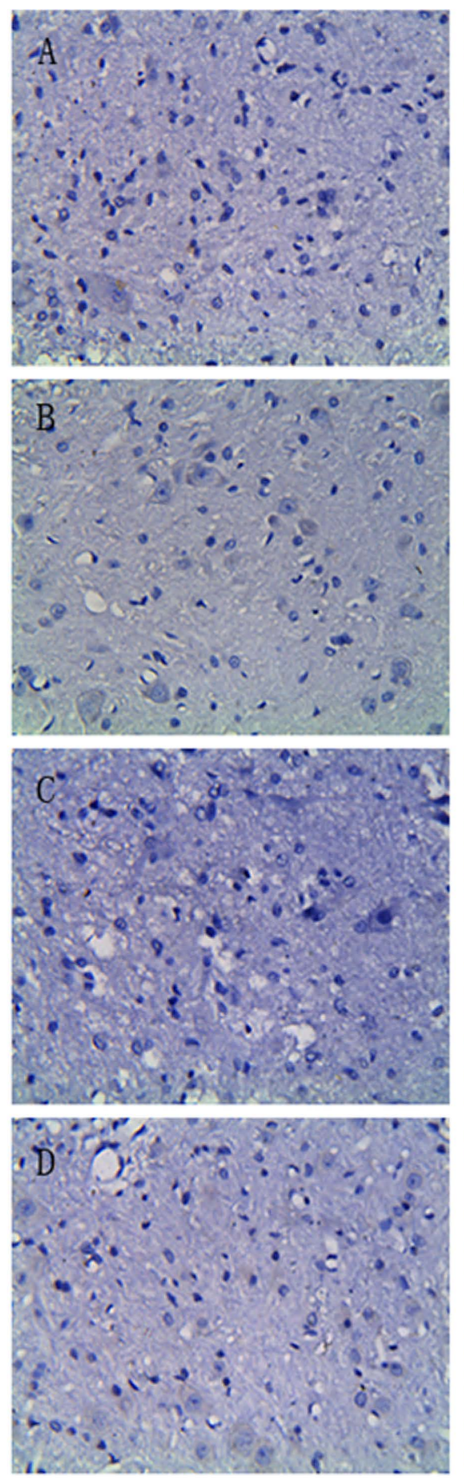

Bcl-2
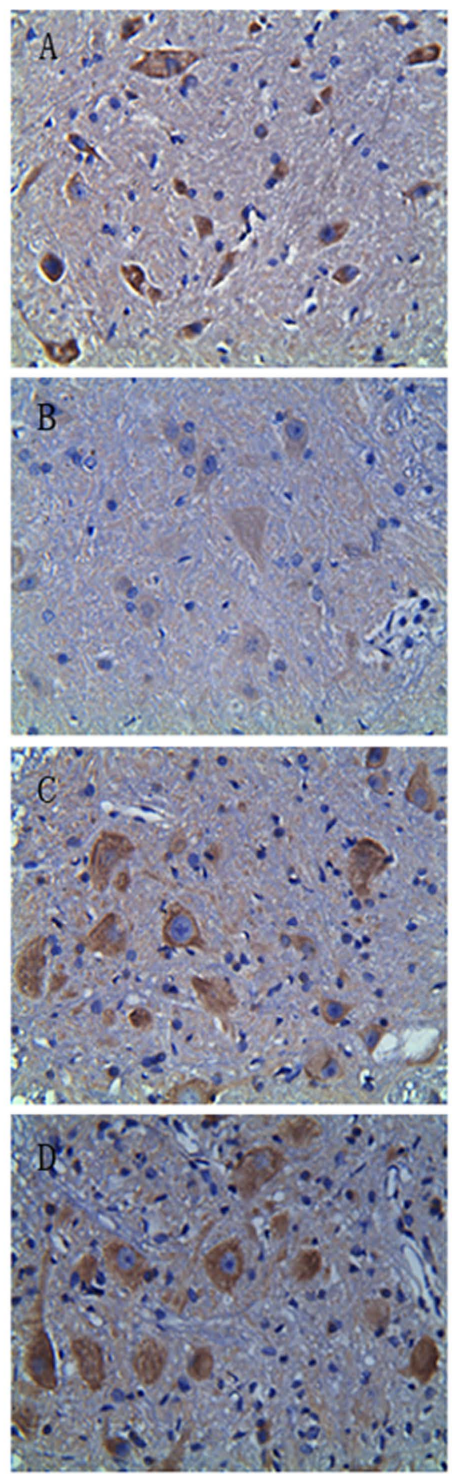

Figure 4. Microscopic observations of Bad, Bax, and Bcl-2 expression in injured spinal cord tissue specimens from rats of different groups at $7 \mathrm{~d}$ post-surgery $(\times 100)$. (A) Sham group; (B) Model group; (C) Electroacupuncture (EA) group; and (D) EA control group. After spinal cord injury, Bad and Bax expression increased, while Bcl-2 expression decreased. EA treatment significantly promoted Bcl-2 expression, but inhibited Bad and Bax expression.

Table 5. The results of immunohistochemical analysis of Bax, Bad, and Bcl-2 in injured spinal cord tissue at $7 \mathrm{~d}$ post-surgery in different groups of rats $(\bar{x} \pm s)$.

\begin{tabular}{|c|c|c|c|}
\hline Group & Bad & Bax & Bcl-2 \\
\hline Sham & $0.06895 \pm 0.012$ & $0.027039 \pm 0.004$ & $0.187429 \pm 0.006$ \\
\hline Model & $0.128251 \pm 0.021$ & $0.150294 \pm 0.003$ & $0.103892 \pm 0.014$ \\
\hline EA & $0.054116 \pm 0.009^{\star \star \star \square}$ & $0.078775 \pm 0.007^{\star \star \omega}$ & $0.173388 \pm 0.004^{\star \star \amalg}$ \\
\hline EA control & $0.071302 \pm 0.004^{\bullet \bullet}$ & $0.117657 \pm 0.018^{\bullet \bullet}$ & $0.145866 \pm 0.005^{\bullet \bullet}$ \\
\hline
\end{tabular}

Note: EA, electroacupuncture; compared with the model group, ${ }^{\star \star} P<0.01,{ }^{\bullet} P<0.05$; and compared with EA control group, ${ }^{\boldsymbol{} "} P<$ 0.05 . 


\section{Discussion}

Presently, SCI is characterized by high mortality, high disability rate, difficult rehabilitation, longer course, and high cost of treatment. SCI prevention, treatment, and rehabilitation have attracted increasing attention in the field of medicine and there is a great need for a simple and efficient means of treatment for this disease. Acupuncture is well known for its simple operation. It is also free of toxic side effects, has a low price, and has proven efficacy. Research shows that acupuncture plays a positive role in neurodegenerative diseases. It can stimulate the release of substances from the central nervous system, such as endorphins, calcitonin gene-related peptides, and neuropeptide Y [16] [17]. Additionally, acupuncture regulates enzyme activity to maintain neuronal self-protective mechanisms [18]. Moreover, it can promote proliferation and differentiation of endogenous neural stem cells in the central nervous system [19]. The present study found that motor nerve function in rats after EA treatment was significantly better than in the model and EA control groups. Additionally, bladder capacity tended to stabilize, with no significant trend towards a decrease in the EA group. These results indicated that the early application of EA stimulation promoted restoration of motor function in rats. However, the protective mechanism of EA for SCI and relevant complications is still unclear. Further studies are needed to better understand the role EA plays in neuronal apoptosis.

Traumatic brain injury, SCI, and stroke are all associated with the occurrence of apoptosis [20]. Crowe et al. [21] first found that the occurrence of apoptosis after SCI caused motor and sensory dysfunction. There is a sequential process of primary and secondary injuries after SCI, and the ultimate extent of the injury is mainly determined by secondary injury initiated by a variety of factors. Apoptosis is an important secondary injury factor that mainly involves the death receptor pathway and mitochondrial signaling pathway. The mitochondrial signaling pathway is the primary pathway for disease development and progression, whereas the Bcl-2 family plays a key role in regulating the execution phase of apoptosis. Common apoptotic genes include Bcl-2, Bax, Bcl-xl, and Bad, among which Bax and $\mathrm{Bcl}-2$ are antagonistic to each other, whereby the $\mathrm{Bcl}-2 / \mathrm{Bax}$ ratio determines the extent of apoptosis [22]. The anti-apoptotic factor Bcl-2 is mainly localized in the mitochondrial outer membrane, endoplasmic reticulum, and nuclear membrane. When cells receive apoptotic stimuli, Bcl-2 and Bcl-xL form heterodimers with the anti-apoptotic protein of the Bcl-2 family through the BH3 domain. This mechanism maintains the localization of pro-apoptotic proteins within the cell and protects cells from undergoing the apoptosis process. Bax and Bad are both pro-apoptotic genes. Increased Bax expression initiates apoptosis possibly through two mechanisms: on one hand, Bax forms a heterodimer with Bcl-2, thereby inhibiting the anti-apoptotic effects of Bcl-2. On the other hand, Bax itself forms a heterodimer to exert a pro-apoptotic effect. Bad is normally localized in the cytoplasm, and when cells are subject to apoptotic stimuli, Bad rapidly dephosphorylates and migrates to the mitochondria, thereby inducing apoptosis [23].

In the present study, a large number of apoptotic neurons were present in spinal cord tissue of the model and treatment groups after SCI. Following EA treatment for $7 \mathrm{~d}$, the number of apoptotic cells in the EA group significantly decreased compared with the model and EA control groups. Additionally, Bcl-2 expression was relatively low after SCI, but significantly increased after $7 \mathrm{~d}$ of EA treatment compared with the model and EA control groups. These results coincided with previous studies [24] [25] and showed that Bax and Bad expression substantially increased after SCI, but decreased after EA intervention. As the key anti-apoptotic and pro-apoptotic factors, increased Bcl-2 expression and drastically reduced Bax and Bad expression may be related to positive and negative regulation of Bcl-2 and Bax. After EA stimulation, Bcl-2 competitively binds to the Bax protein to form a stable Bax/Bcl-2 heterodimer, counteracting Bax/Bax-induced apoptosis. Bad can bind to Bcl-2 and Bcl-xl to form heterodimers. However, after EA stimulation, p-Bad can bind to the chaperone protein 14-3-3 and thus interfere with the binding of Bad to Bcl-2 and Bcl-xl, further inhibiting apoptosis. Therefore, mutual regulation of Bcl-2, Bax, and Bad proteins can inhibit neuronal apoptosis after SCI, whereas EA treatment promotes Bcl-2 expression and reduces Bax and Bad expression. This may be one of the reasons why EA inhibits injured spinal cord apoptosis and promotes spinal cord repair.

\section{Conclusion}

In conclusion, this study demonstrated that EA treatment at the Zhibian and Shuidao acupoints significantly improved relevant functions after SCI. One of the possible mechanisms is that EA treatment inhibits expression of pro-apoptotic genes Bax and Bad, but promotes expression of anti-apoptotic gene Bcl-2. However, the occurrence of apoptosis is a complex regulatory process. It includes three stages of gene regulation, signal transduc- 
tion, and apoptotic execution, respectively, ultimately eliminating unnecessary or abnormal cells. Activation of the signal transduction pathway during the early stages of apoptosis is a necessary precondition for the occurrence of apoptosis and has been increasingly used in studies of neurological diseases in recent years. Nevertheless, further studies are needed to determine the exact mechanism underlying the promotion of spinal cord injury repair by EA treatment.

\section{Acknowledgements}

This study was supported by the Zhejiang Chinese Medical Science Research Foundation (No. 2008CB067).

\section{References}

[1] Xia, L.P., Fan, F., Tang, A.L. and Ye, W.Q. (2014) Effects of Electroacupuncture Combined with Bladder Training on the Bladder Function of Patients with Neurogenic Bladder after Spinal Cord Injury. International Journal of Clinical and Experimental Medicine, 7, 1344-1348.

[2] Fischer, M.J., Krishnamoorthi, V.R., Smith, B.M., Evans, C.T., St Andre, J.R., Ganesh, S., et al. (2012) Prevalence of Chronic Kidney Disease in Patients with Spinal Cord Injuries/Disorders. American Journal of Nephrology, 36, 542548. http://dx.doi.org/10.1159/000345460

[3] Xing, G.G., Liu, F.Y., Qu, X.X., Han, J.S. and Wan, Y. (2007) Long-Term Synaptic Plasticity in the Spinal Dorsal Horn and Its Modulation by Electroacupuncture in Rats with Neuropathic Pain. Experimental Neurology, 208, 323-332. http://dx.doi.org/10.1016/j.expneurol.2007.09.004

[4] Hwang, L., Choi, I.Y., Kim, S.E., Ko, I.G., Shin, M.S., Kim, C.J., et al. (2013) Dexmedetomidine Ameliorates Intracerebral Hemorrhage-Induced Memory Impairment by Inhibiting Apoptosis Andenhancing Brain-Derived Neurotrophic Factor Expression in the Rat Hippocampus. International Journal of Molecular Medicine, 31, 1047-1056.

[5] Sung, Y.H., Kim, S.C., Hong, H.P., Park, C.Y., Shin, M.S., Kim, C.J., et al. (2012) Treadmill Exercise Ameliorates Dopaminergic Neuronal Loss through Suppressing Microglial Activation in Parkinson's Disease Mice. Life Sciences, 91, 1309-1316. http://dx.doi.org/10.1016/j.lfs.2012.10.003

[6] Okuno, S., Saito, A., Hayashi, T. and Chan, P.H. (2004) The c-Jun N-Terminal Protein Kinase Signaling Pathway Mediates Bax Activation and Subsequent Neuronal Apoptosis through Interaction with Bim after Transient Focal Cerebral Ischemia. Journal of Neuroscience, 24, 7879-7887. http://dx.doi.org/10.1523/JNEUROSCI.1745-04.2004

[7] Chen, M.H., Ren, Q.X., Yang, W.F., Chen, X.L., Lu, C. and Sun, J. (2013) Influences of HIF-l $\alpha$ on Bax/Bcl-2 and VEGF Expressions in Rats with Spinal Cord Injury. International Journal of Clinical and Experimental Pathology, 6, 2312-2322.

[8] Li, Y., Gu, J., Liu, Y., Long, H., Wang, G., Yin, G., et al. (2013) iNOS Participates in Apoptosis of Spinal Cord Neurons via p-BAD Dephosphorylation Following Ischemia/Reperfusion (I/R) Injury in Rat Spinal Cord. Neuroscience Letters, 545, 117-122. http://dx.doi.org/10.1016/j.neulet.2013.04.043

[9] Sun, W., Winseck, A., Vinsant, S., Park, O.H., Kim, H. and Oppenheim, R.W. (2004) Programmed Cell Death of Adult-Generated Hippocampal Neurons Is Mediated by the Proapoptotic Gene Bax. Journal of Neuroscience, 24, 11205-11213. http://dx.doi.org/10.1523/JNEUROSCI.1436-04.2004

[10] Norrbrink, C. and Lundeberg, T. (2011) Acupuncture and Massage Therapy for Neuropathic Pain Following Spinal Cord Injury: An Exploratory Study. Acupuncture in Medicine, 29, 108-115. http://dx.doi.org/10.1136/aim.2010.003269

[11] Pei, J., Sun, L., Chen, R., Zhu, T., Qian, Y. and Yuan, D. (2001) The Effect of Electro-Acupuncture on Motor Function Recovery in Patients with Acute Cerebral Infarction: A Randomly Controlled Trial. Journal of Traditional Chinese Medicine, 21, 270-272.

[12] Lin, H.S., Ji, Z.S., Zheng, L.H., Guo, G.Q., Chen, B., Zhang, G.W. and Wu, H. (2012) Effect of Methylprednisolone on the Activities of Caspase-3, -6, -8 and -9 in Rabbits with Acute Spinal Cord Injury. Experimental and Therapeutic Medicine, 4, 49-54.

[13] Zhou, Y., Liu, X.H., Qu, S.D., Yang, J., Wang, Z.W., Gao, C.J., et al. (2013) Hyperbaric Oxygen Intervention on Expression of Hypoxia-Inducible Factor- $1 \alpha$ and Vascular Endothelial Growth Factor in Spinal Cord Injury Models in Rats. Chinese Medical Journal (English), 126, 3897-3903.

[14] Rajan, R.K., Juler, G. and Eltorai, I.M. (2000) Sigmoid Colon Rupture Secondary to Credé’s Method in a Patient with Spinal Cord Injury. The Journal of Spinal Cord Medicine, 23, 90-91.

[15] Nomura, S., Ishido, T., Teranishi, J. and Makiyama, K. (2000) Long-Term Analysis of Suprapubic Cystostomy Drainage in Patients with Neurogenic Bladder. Urologia Internationalis, 65, 185-189. http://dx.doi.org/10.1159/000064873

[16] Feng, X.D., Yang, S.L., Liu, J., Huang, J., Peng, J., Lin, J.M., et al. (2013) Electroacupuncture Ameliorates Cognitive 
Impairment through Inhibition of NF- $\kappa \mathrm{B}-$ Mediated Neuronal Cell Apoptosis in Cerebral Ischemia-Reperfusion Injured Rats. Molecular Medicine Reports, 7, 1516-1522.

[17] Lee, B., Sur, B., Shim, J., Hahm, D.H. and Lee, H. (2014) Acupuncture Stimulation Improves Scopolamine-Induced Cognitive Impairment via Activation of Cholinergic System and Regulation of BDNF and CREB Expressions in Rats. BMC Complementary and Alternative Medicine, 14, 338.

[18] Cha, M.H., Bai, S.J., Lee, K.H., Cho, Z.H., Kim, Y.B., Lee, H.J., et al. (2010) Acute Electroacupuncture Inhibits Nitric oxide Synthase Expression in the Spinal Cord of Neuropathic Rats. Neurological Research, 32, 96-100. http://dx.doi.org/10.1179/016164109X12537002794363

[19] Liu, Z., Ding, Y. and Zeng, Y.S. (2011) A New Combined Therapeutic Strategy of Governor Vessel Electro-Acupuncture and Adult Stem Cell Transplantation Promotes the Recovery of Injured Spinal Cord. Current Medicinal Chemistry, 18, 5165-5171. http://dx.doi.org/10.2174/092986711797636144

[20] Kim, D.H., Ko, I.G., Kim, B.K., Kim, T.W., Kim, S.E., Shin, M.S., et al. (2010) Treadmill Exercise Inhibits Traumatic Brain Injury-Induced Hippocampal Apoptosis. Physiology \& Behavior, 101, 660-665. http://dx.doi.org/10.1016/j.physbeh.2010.09.021

[21] Crowe, M.J., Bresnahan, J.C., Shuman, S.L., Masters, J.N. and Beattie, M.S. (1997) Apoptosis and Delayed Degeneration after Spinal Cord Injury in Rats and Monkeys. Nature Medicine, 3, 73-76.

[22] Hou, Q., Cymbalyuk, E., Hsu, S.C., Xu, M. and Hsu, Y.T. (2003) Apoptosis Modulatory Activities of Transiently Expressed Bcl-2: Roles in Cytochrome $c$ Release and Bax Regulation. Apoptosis, 8, 617-629. http://dx.doi.org/10.1023/A:1026187526113

[23] Zhang, X.H., Chen, S.Y., Tang, L., Shen, Y.Z., Luo, L., Xu, C.W., et al. (2013) Myricetin Induces Apoptosis in HepG2 Cells through Akt/p70S6K/Bad Signaling and Mitochondrial Apoptotic Pathway. Anti-Cancer Agents in Medicinal Chemistry, 13, 1575-1581. http://dx.doi.org/10.2174/1871520613666131125123059

[24] Bleicken, S., Wagner, C. and Garcia-Saez, A.J. (2013) Mechanistic Differences in the Membrane Activity of Bax and Bcl-xL Correlate with Their Opposing Roles in Apoptosis. Biophysical Journal, 104, 421-431. http://dx.doi.org/10.1016/j.bpj.2012.12.010

[25] Wang, T., Liu, C.Z., Yu, J.C., Jiang, W. and Han, J.X. (2009) Acupuncture Protected Cerebral Multi-Infarction Rats from Memory Impairment by Regulating the Expression of Apoptosis Related Genes Bcl-2 and Bax in Hippocampus. Physiology \& Behavior, 96, 155-161. http://dx.doi.org/10.1016/j.physbeh.2008.09.024 
Scientific Research Publishing (SCIRP) is one of the largest Open Access journal publishers. It is currently publishing more than 200 open access, online, peer-reviewed journals covering a wide range of academic disciplines. SCIRP serves the worldwide academic communities and contributes to the progress and application of science with its publication.

Other selected journals from SCIRP are listed as below. Submit your manuscript to us via either submit@scirp.org or Online Submission Portal.
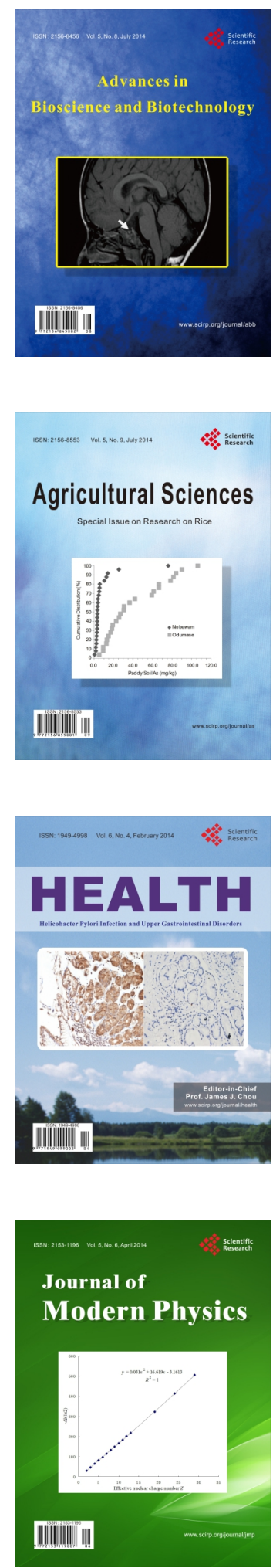
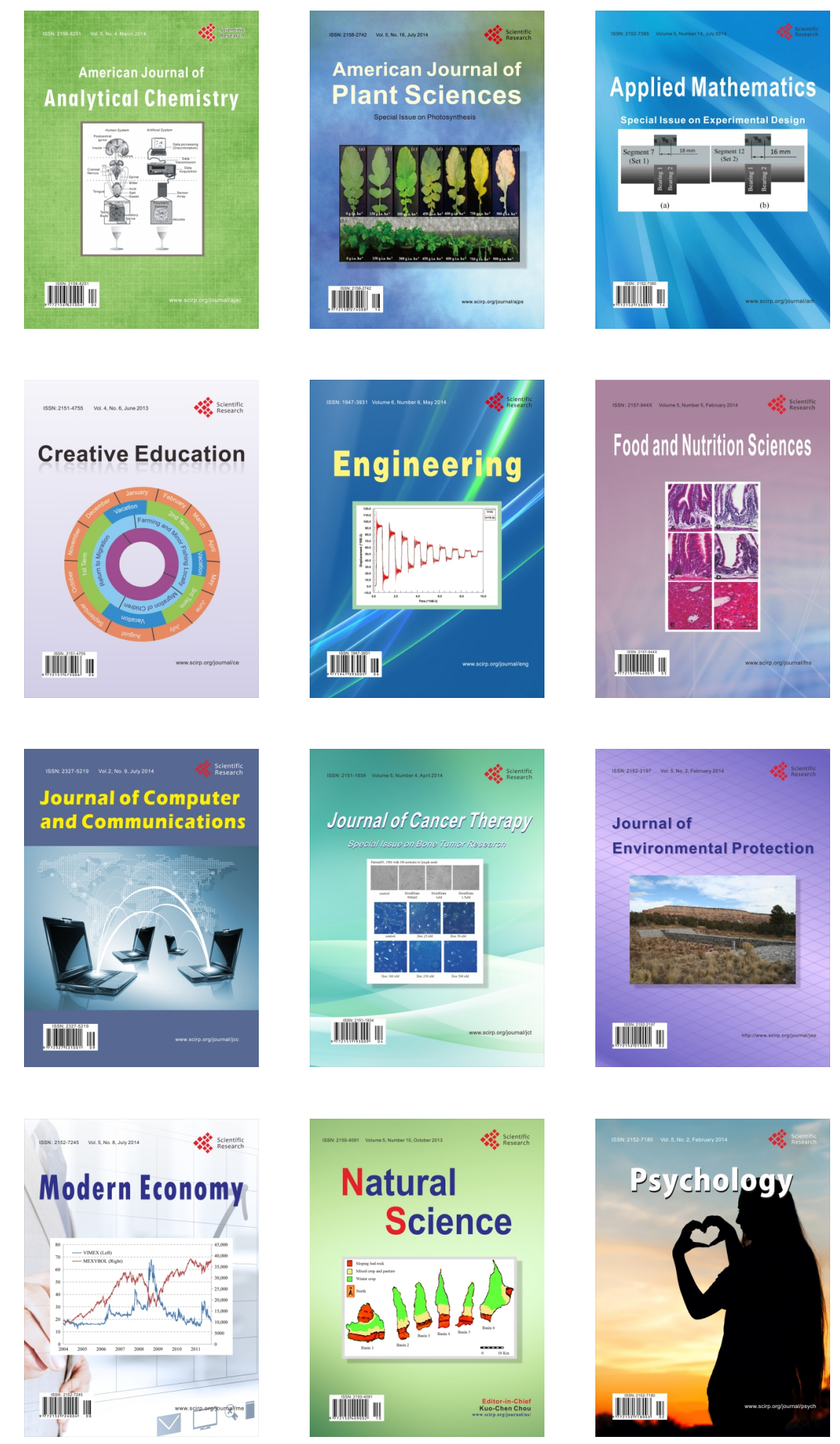